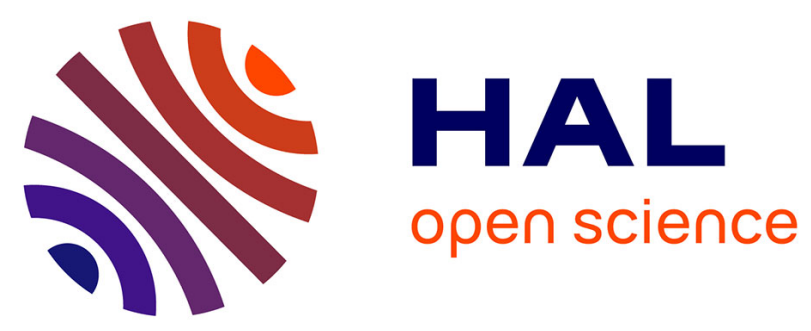

\title{
Search for non-Gaussian events in the data of the VIRGO E4 engineering run
}

\author{
F. Acernese, P. Amico, N. Arnaud, D. Babusci, G. Ballardin, R. Barilli, F. \\ Barone, M. Barsuglia, F. Beauville, F. Bellachia, et al.
}

\section{- To cite this version:}

F. Acernese, P. Amico, N. Arnaud, D. Babusci, G. Ballardin, et al.. Search for non-Gaussian events in the data of the VIRGO E4 engineering run. Gravitational Wave Data Analysis Workshop 7, 2003, Kyoto, Japan. pp.623-632. in2p3-00014167

\section{HAL Id: in2p3-00014167 https://hal.in2p3.fr/in2p3-00014167}

Submitted on 25 Nov 2003

HAL is a multi-disciplinary open access archive for the deposit and dissemination of scientific research documents, whether they are published or not. The documents may come from teaching and research institutions in France or abroad, or from public or private research centers.
L'archive ouverte pluridisciplinaire HAL, est destinée au dépôt et à la diffusion de documents scientifiques de niveau recherche, publiés ou non, émanant des établissements d'enseignement et de recherche français ou étrangers, des laboratoires publics ou privés. 


\title{
Search for non-gaussian events in the data of Virgo E4 engineering run
}

\author{
The VIRGO Collaboration $\nmid$ (presented by Didier Verkindt $\ddagger$ ) \\ † Traversa H di via Macerata, 56021 San Stefano a Macerata, Cascina (Pisa), Italy. \\ $\ddagger$ LAPP, Chemin de Bellevue, 74941 Annecy-le-Vieux \\ E-mail: verkindt@lapp.in2p3.fr
}

\begin{abstract}
This work is a first attempt to search for glitch events in the data of the Virgo Central Interferometer (CITF). In a first step, using simple algorithms, we have searched for strong glitches in the interferometer's output photodiodes signals, over the 3 days of data of the last engineering run. In a second step, we have focused on 10 hours of data while the interferometer was locked and stable. The events found in the dark fringe signal during this period are all within $3 \mathrm{rms}$ around the mean output value of each algorithm and are, in some cases, linked to a beat between the powerline $50 \mathrm{~Hz}$ contamination and a $43.7 \mathrm{~Hz}$ line that comes from the injection system components. The main conclusions are that, at this level of analysis, the algorithms used have found no strong glitch-like events in the dark fringe signal of Virgo and that removing spectral lines like the $50 \mathrm{~Hz}$ is essential to investigate further.
\end{abstract}

Submitted to: Class. Quantum Grav. 


\section{Introduction}

VIRGO is a long baseline interferometer aimed at the detection of gravitational waves. During the years 2001 and 2002, a preliminary version of the VIRGO interferometer [1], with arms of 6 meters long and called CITF (Central InTerFerometer), has been commissioned $[2,3]$. During this commissioning a search for glitches has been prepared in order to find events that should be vetoed in the futur and in order to exercise detector monitoring software tools.

From september 2001 to july 2002, several commissioning engineering runs have been undertaken. Each time, a search for glitches was performed on the data recorded. Run E4 was the last run and lasted 3 days, from 12th to 15th of July 2002. During E4, most of the components and most of the control loops of the future $3 \mathrm{~km}$ Virgo detector were active. About 1 TBytes of data were recorded and, before the finalization of the Virgo online monitoring, we performed an off-line search for glitches, mainly in the photodiodes channels. The aim was to find detector noises that could be removed by hardware or software modifications and to have a first look at the detector's fake signals that could prevent a search for astrophysical events.

We present here the first results obtained: some examples of strong glitches found and a study of the dark fringe signal during a stable period of 10 hours. More detailed results of this analysis are presented in a Virgo note [4].

\section{Algorithms used}

Two algorithms were used for this search of glitches. Both work in the time domain and are derived from one algorithm developed for the Virgo online monitoring [5]. The first one (algo1) take as input the signal itself, the second one (algo2) includes a differenciation step (simplest high-pass filter) and works then on the signal derivative.

Each algorithm uses a running mean and a running rms of the signal, which are computed using a sliding window and an exponentially decreasing weight $w=$ $\exp (-1 / \tau)$, where $\tau$ is expressed in number of samples. In order for the algorithms to be quite sensitive to local variations without being too much non-linear, we have used $\tau=40$ samples (corresponding to a time window of $2 \mathrm{~ms}$ for a signal sampled at $20 \mathrm{kHz})$.

If $m_{i}$ and $r_{i}$ are the running mean and running rms computed on the quantity $x_{i}$ which is the photodiode's signal (input of algo1) or its derivative (input of algo2), the output $s_{i}$ of the algorithm is defined by:

$$
s_{i}=\left(x_{i}-m_{i}\right) / r_{i} \quad \text { with } \quad m_{i}=\frac{1}{N} \Sigma_{j} x_{j} w^{i-j} \quad \text { and } \quad r_{i}=\left(\frac{1}{N} \Sigma_{j} x_{j}^{2} w^{i-j}-m_{i}^{2}\right)^{1 / 2}
$$

where $\mathrm{N}$ is the sum of the weights $\left(N=\Sigma_{j} w^{i-j}\right)$. The algorithm triggers if $\left|s_{i}\right|$ is greater than a user defined threshold.

In order to get meaningful results, before passing the data to both algorithms we check 
that the interferometer is locked on dark fringe and that the output mode-cleaner is locked on TEM00 mode.

Both algorithms were used offline, with the help of the GAI online monitoring tool [6] or using a macro written in $\mathrm{C}$, in the Vega environment [7]. One example of glitch found by the two algorithms is shown on figure 1 .

Even with the chosen parameter $\tau=40$ samples, the non-linearity of the algorithms (due to the running mean and rms), prevent to express the thresholds as a number of standard deviations above a mean. Such thresholds are compared to the number of running rms above the running mean. So, the choice of the thresholds was based on the known strong glitches observed (like the one of figure 2) instead of statistical considerations.

\section{Examples of loudest glitches seen in photodiodes signals}

Some large glitches were found by the algorithms in run E4, when using a high threshold (threshold $=6$ for algo1, threshold $=10$ for algo 2 ). The algorithms were used on the dark fringe signal before the output mode cleaner (B1p), on the dark fringe signal after the output mode cleaner (B1) and on the recycled power signal (B5). For each type of glitch, we tried to identify the source and, when it was needed, hardware or software modifications were decided to correct or eliminate this source. The full classification of the loudest glitches found in E4 and in previous runs is available in [4].

\section{Events related to the electronics:}

In the engineering runs before E4, the algorithms found electronics glitches that were linked to ADC problems. Hardware modifications were done to eliminate such problems. Then, during E4, the algorithms still found small glitches in the B1p signals, a few ADC counts high and one sample long, that can be related to the photodiode's digital electronics (see figure 1).

\section{Events related to alignement or calibration procedures:}

Other glitches have been related to remote actions on the components of the interferometer. One example is shown on figure 2. We have found large oscillations simultaneously on all B1 channels. The frequency of these oscillations is around $1800 \mathrm{~Hz}$, they are about $50 \mathrm{~ms}$ long and there are several of them over a few seconds. It turned out that these glitches are all simultaneous to a realignment of the detection bench telescope. This alignment is performed with motors acting on the mirrors of the telescope (picomotors activated at a frequency of $1800 \mathrm{~Hz}$ ).

\section{Events related to human actions:}

Other types of human actions are at the origin of some events detected. One example is the switch between dark fringe signals (B1p or B1) used to lock the interferometer (see figure 3). During E4, first lock on dark fringe was acquired using B1p, then the output 
Mode Cleaner was locked and B1 was used for locking instead of B1p. Since there are more modes in B1p than in B1 and since electronics gains for B1 and B1p photodiodes are not identical, the level is not necessarily the same at the output of B1 and B1p photodiodes. This results in a glitch on dark fringe correction signal and a change of level on B1 and B1p signals, at the time the switch was done. About 10 such glitches characterized by a rapid change of level on B1 and B1p dark fringe signals have been observed during E4. In the future, the situation will be improved by setting a smoother switch between B1 and B1p.

If only stable periods of E4 are analysed, which means when the interferometer is fully locked on B1 and there is no alignment, calibration, or human activity in the central building, the only glitches found are the small glitches found on B1p and related to the electronics.

\section{Study of the dark fringe signal over a stable period of 10 hours}

We choose to focus on a stable period of the interferometer: 10 hours from GPS=710551143 (Fri Jul 12 23:19:03 2002 UTC) to GPS=710558743 (Fri Jul 13 09:19:03 2002 UTC) and to focus on the demodulated photodiode dark fringe signal after the output mode-cleaner (B1_ACq channel).

First, in order to exclude any data containing glitches from picomotors activation, we checked that picomotors are not active. This excludes 218 seconds from the 36000 seconds of data analysed. On the remaining data, we looked at the signal distribution and we used the algorithms.

\subsection{Tails of the B1_ACq distribution}

Looking straightforwardly at the evolution of the signal B1_ACq distribution, we obtained the figure 4 that shows the time evolution of the B1_ACq signal distribution and the B1_ACq signal derivative distribution, for the 10 hours data set. In those two plots, each vertical colored line represents the signal distribution over 10 seconds (200000 samplings). It allows to follow the evolution of the signal distribution and to see where the tails are built. In those plots, two parts can be isolated:

- During about 30 seconds, from $\mathrm{GPS}=710556730$ to $\mathrm{GPS}=710556790$, an action made on the input Mode-Cleaner created a high amplitude $36 \mathrm{~Hz}$ noise on all the photodiodes channels. This can be seen on the plots of figure 4 around the value 5600 as large tails generated in the signal distribution. We will see in section 4.4 that this noise, even if not of glitch type, was "detected" by the "glitch search" algorithms.

- The last 3000 seconds contain noisier data. This is a consequence of the closing of the angular alignment loop of the input mode cleaner, at GPS $=710584258$. We 
will see in section 4.2 that the algorithm algo 2 was sensitive to this noise increase in the derivative of the input signal.

\subsection{Search for glitch events in the dark fringe signal}

Looking at the data with the algorithms, we have found in the B1_ACq signal 8 events above a threshold of 3.5 for algo1 and 30 events above a threshold of 5.5 for algo2 (from which 17 events are in the noisier last hour of the data set). The two algorithms have their events at different times because algo1 is sensitive to the changes of the signal and algo2 is sensitive to the changes of the derivative of the signal. For large glitches (see section 3), their answers are similar. Figure 5 shows, for each algorithm, the largest event found in the 10 hours of data.

The origin of the largest event seen by algo1 is a beat phenomenon between the $50 \mathrm{~Hz}$ contamination and a $43.7 \mathrm{~Hz}$ line that is coherent with the input Mode Cleaner angular correction signal, that provides a "quiet zone" of data every 0.2 second (see figure 7). In such a "quiet zone", the running rms computed by algo1 is reduced and the algorithm gives then a high output at the exit of the zone (the "event" detected is at sample 14755 on upper plot of figure 5).

As can be seen on lower plot of figure 5, at this level of analysis, there is no indication that the events found by algo2 have a physical source (it may be just statistical fluctuation in the B1_ACq dark fringe signal). Using the derivative of the signal protects relatively this algorithm from the $43.7 \mathrm{~Hz}-50 \mathrm{~Hz}$ beat phenomenon seen by algo1.

Figure 6 shows the maximum per second of the output of each algorithm versus the GPS time. Except for the presence of noisier data in the last hour, no structure in time is visible at the output of the two algorithms, over the 10 hours of data analysed.

\subsection{Down-sampling of the B1_ACq signal}

For both algorithms, a down-sampling of the data at $2 \mathrm{kHz}$ has also been performed in order to look at larger time scale glitches in B1_ACq. Running the algorithms on the down-sampled data, we have found 31 events above a threshold of 3.5 for algo1 (from which 18 events are in the noisier last hour of data) and 24 events above a threshold of 4 for algo2 (from which 7 events are in the noisier last hour of data).

Figure 8 shows the strongest events found by each algorithm, after down-sampling at $2 \mathrm{kHz}$, in the 10 hours of data. The beat between the $43.7 \mathrm{~Hz}$ and $50 \mathrm{~Hz}$ lines or some statistical fluctuations are, once again, at the origin of those events.

\subsection{The $36 \mathrm{~Hz}$ noise at GPS=710556730}

Figure 9 shows, in a time and a time-frequency plots, the 30 seconds of data from GPS $=710556730$ to $\mathrm{GPS}=710556790$, where a $36 \mathrm{~Hz}$ noise appeared after acting on the alignment control of the input mode cleaner. Figure 10 is a zoom of figure 6 showing that, during these 30 seconds, the output of both algorithms are minimal (because the 
low frequency $36 \mathrm{~Hz}$ signal saturates any other fluctutations of B1_ACq and the running mean follows well the $36 \mathrm{~Hz}$ sinusoide). The algorithms are sensitive to spectrum changes like dominant peaks appearing in the spectrum and they have "detected" the $36 \mathrm{~Hz}$ noise by a decrease of their output over the 30 seconds noise duration. 


\section{Conclusions}

Strong glitches have been found in photodiodes signals, during the Virgo CITF engineering runs, and most of them were due to human actions on the interferometer. Most of the loudest glitches due to photodiode electronics were removed by a recabling of the ADC boards. A softer switch between B1p and B1 photodiodes used for dark fringe loop will be implemented. Full automatic alignement will reduce in the futur the number of manual alignments (use of picomotors) while the interferometer is locked.

In the E4 run data, a 10 hours clean and stable period (interferometer locked, output Mode Cleaner locked and no manual alignment performed) was analysed and no strong glitch was found by our algorithms in the dark fringe signal after the output Mode Cleaner. Some of the largest events found were due to a beat between the $50 \mathrm{~Hz}$ power line contamination and a $43.7 \mathrm{~Hz}$ line that comes from one of the injection system components.

Down-sampling of the data has been used to detect some events at longer timescale. In the present state of the analysis, the events found can be associated with statistical fluctuations or, once again, with the beat between $50 \mathrm{~Hz}$ and $43.7 \mathrm{~Hz}$ lines.

At this level of analysis, no strong "glitch-like" event has been seen in the dark fringe signal of Virgo's CITF. Removing the powerline $50 \mathrm{~Hz}$ contamination (and other low frequency noises like the $43.7 \mathrm{~Hz}$ line) from the dark fringe signal is essential to investigate further. 


\section{References}

[1] Virgo Status

"Status of Virgo", Virgo Collaboration (presented by A. Vicere), GWDAW 2002 proceedings.

[2] Commissioning of the Central Interferometer of the Gravitational Waves Interferometer Virgo. Virgo Collaboration, to be published in Classical and Quantum Gravity.

[3] Central Interferometer Commissioning. Final Report

M. Barsuglia, F. Bondu, R. Flaminio, P. La Penna, G. Losurdo, E. Majorana;

Virgo Note VIR-NOT-LAP-1390-224, 15 Oct. 2002

See also http://www.virgo.infn.it

[4] Search for glitches events in E2, E3 and E4 runs

E. Tournefier, D. Verkindt; Virgo Note VIR-NOT-LAP-1390-230, 15 Jan. 2003

See also http://www.virgo.infn.it or http://wwwlapp.in2p3.fr/virgo

[5] Detector Monitoring Algorithms in the General Algorithm Interface tool (GAI).

A. Kaczmarska; Virgo Note VIR-MAN-LAP-5400-118

[6] General Algorithm Interface (GAI). Software tool for online monitoring.

D. Verkindt; VIR-MAN-LAP-5400-117.

See also documentation at http://wwwlapp.in2p3.fr/virgo/gai

[7] Vega, Visual Environment for Gravitational waves data Analysis.

D. Buskulic, International Journal of Modern Physics D, Vol. 9, 3 (2000) p.293

A software environment based on Root.

See also documentation at http://wwwlapp.in2p3.fr/virgo/vega 


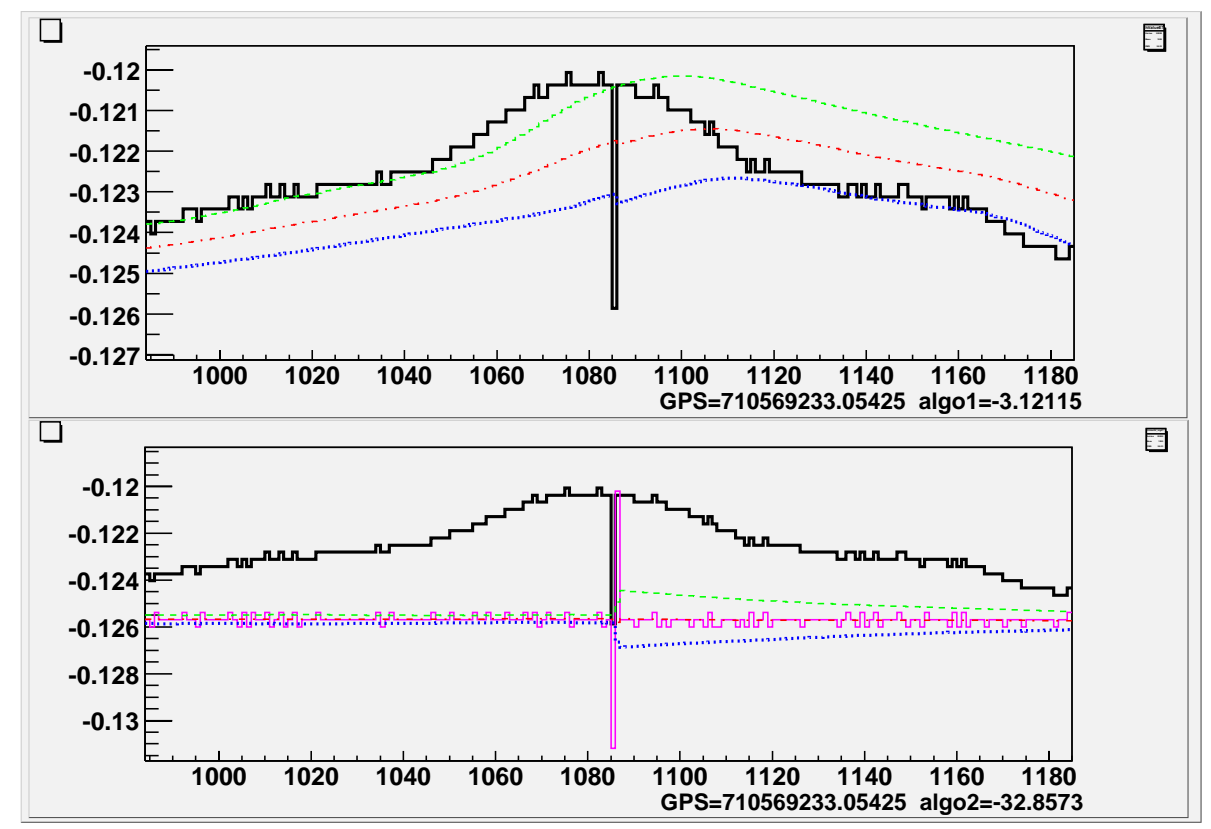

Figure 1. Example of glitch seen by the algorithms. The lower figure shows an event found by algo2 (a glitch seen only on the B1p_ACq dark fringe signal, at GPS=710569233.05, that may be due to a problem in photodiode numerization electronics): the pink thin, red semi-dashed, blue dotted and green dashed lines are respectively the algo2 $x_{i}, m_{i}, m_{i}-r_{i}$ and $m_{i}+r_{i}$ values. The upper figure shows the same event seen by algo1: the red semi-dashed, blue dotted and green dashed lines are respectively the algo $1 m_{i}, m_{i}-r_{i}$ and $m_{i}+r_{i}$ values. Vertical axis is in arbitrary units.

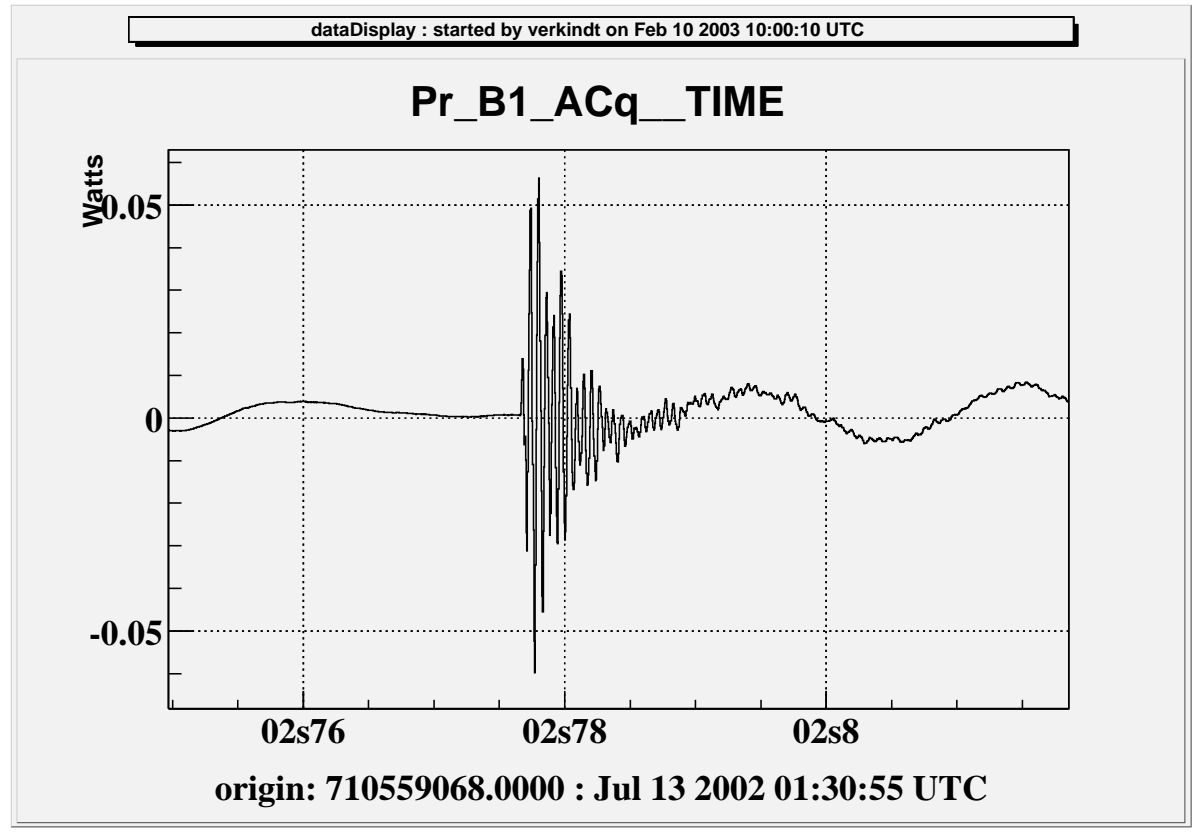

Figure 2. Example of glitch seen on the dark fringe signal and correlated with the manual alignment of the interferometer (use of picomotors). Horizontal axis is time in seconds (0s8 mean 0.8 second). 


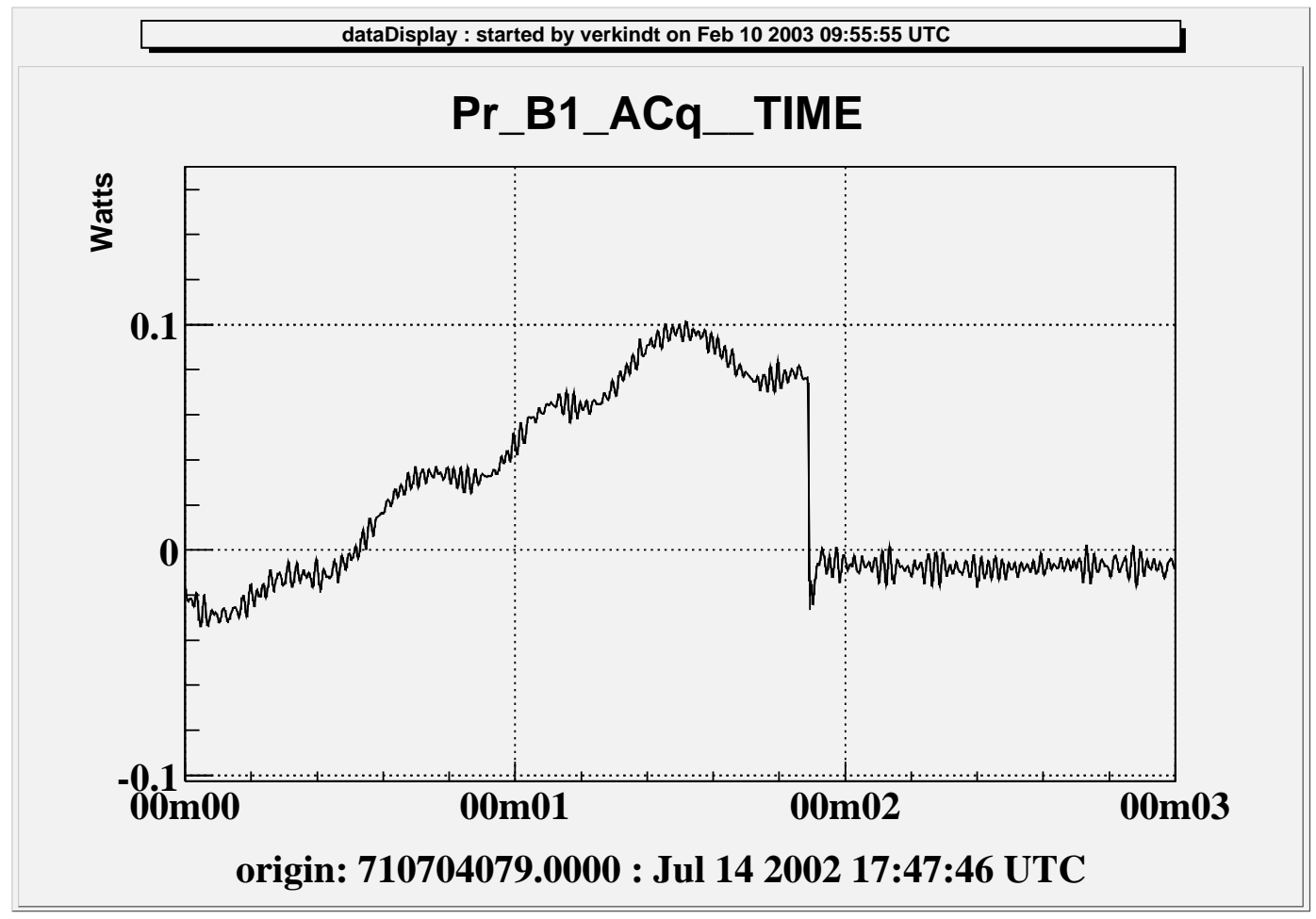

Figure 3. Example of glitch seen on the dark fringe signal and correlated with a human action: the switch between the dark fringe signals B1p and B1 used to lock the interferometer. Horizontal axis is time in minutes and seconds (0m40 mean 0 minutes and 40 seconds).

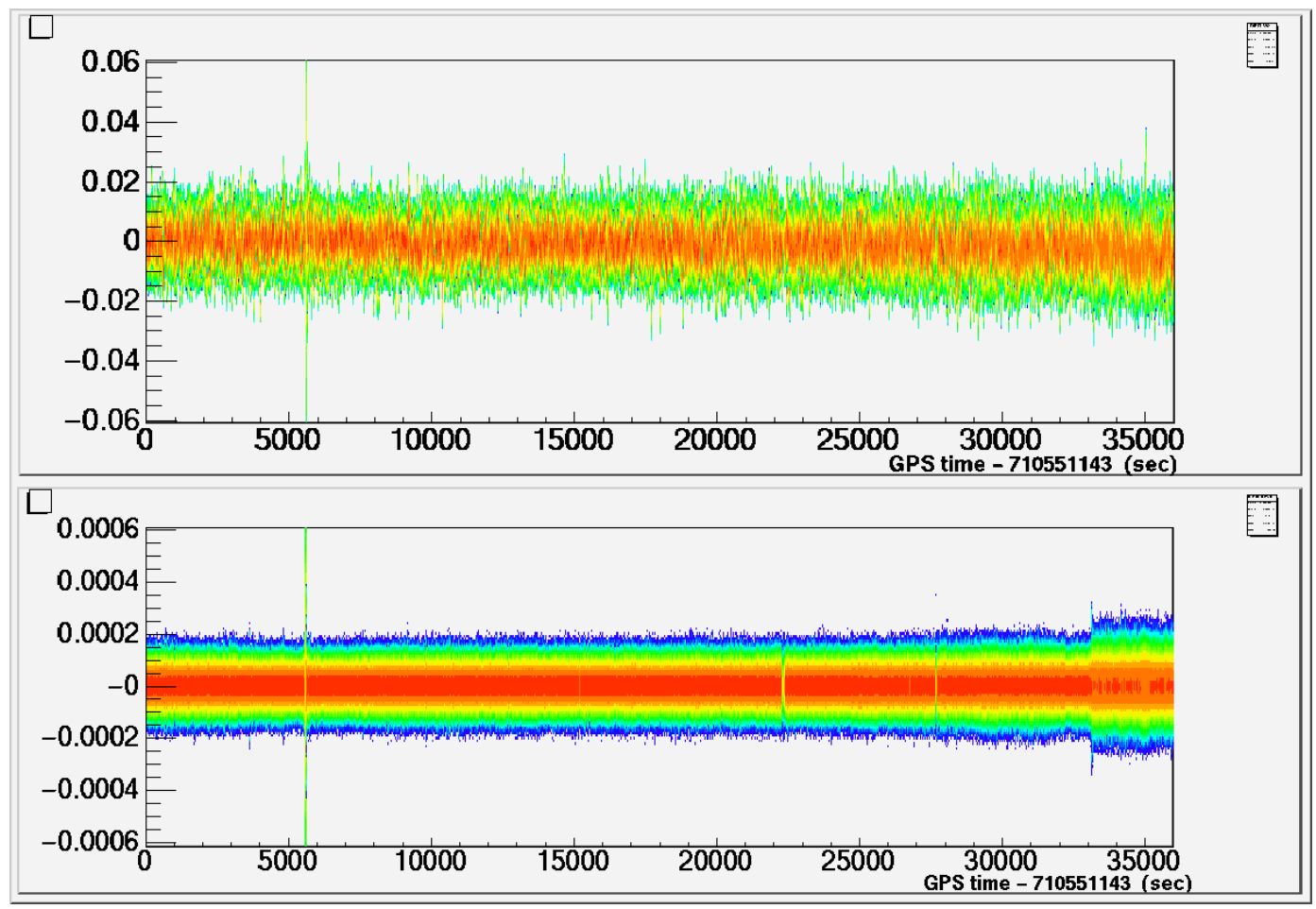

Figure 4. Time evolution of the dark fringe signal distribution (upper plot) and of its derivative (lower plot). Each vertical line contains the distribution of the signal over 10 seconds of data (200000 samples). Vertical axis is in arbitrary units. The color scale does not matter since we are interested in just looking at the extension on the vertical axis with time. 


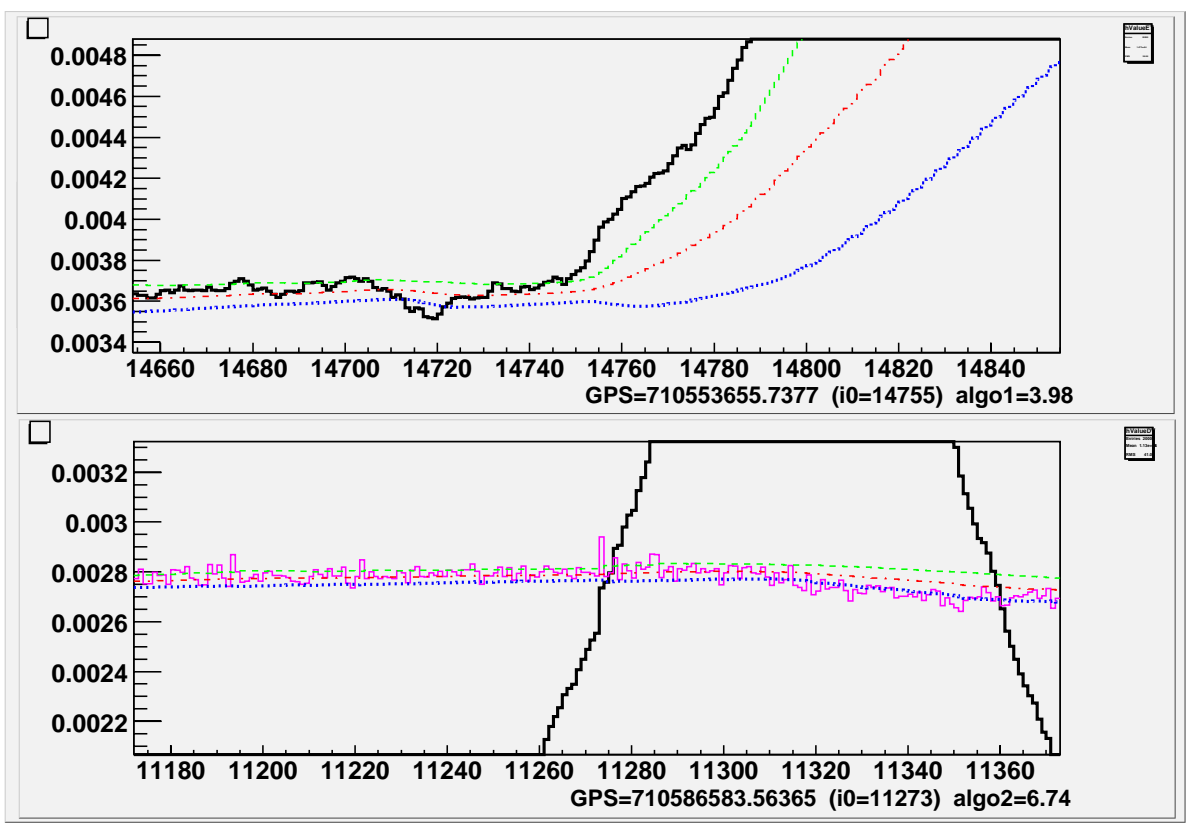

Figure 5. The largest events seen by algo1 and algo2 in the 10 hours of data. [algo1 maximum: 3.98 at GPS=710553655.7377] [algo2 maximum: 6.74 at GPS=710586583.5636]. Colored lines have the same meaning as in figure 1. On upper plot algo1 has triggered on sample 14755, while exiting of a quiet zone of a beat phenomenon. On lower plot algo2 has triggered on sample 11273, which seems to be just a statistical fluctuation of the signal's derivative. Vertical axis is in arbitrary units.

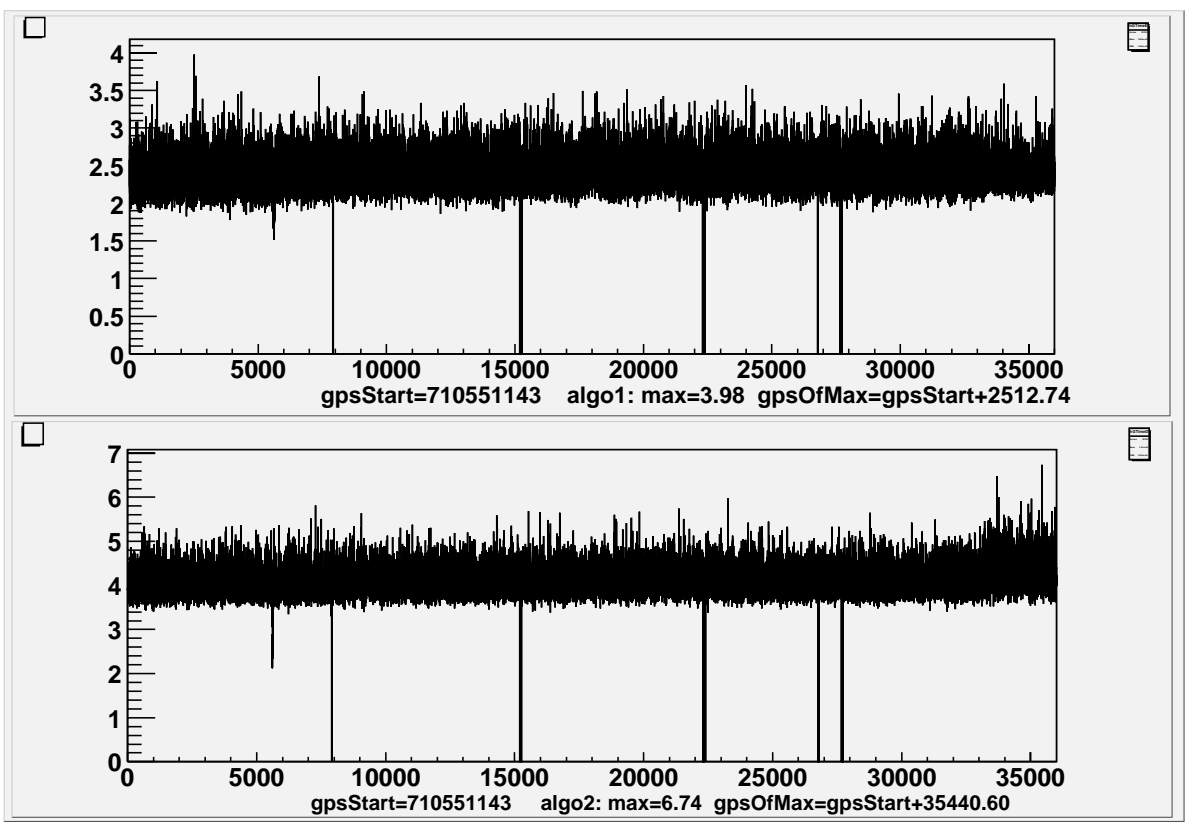

Figure 6. The maximum per second of the algorithm's output absolute value versus GPS time. Upper plot is for algo1 and lower plot is for algo2. Vertical line falling to zero are the part of data vetoed when manual alignments were performed on the interferometer (picomotors active). Largest events are located in the last hour of the data set for algo2 and in the first two hours of the data set for algo1. Vertical axis is in arbitrary units. 


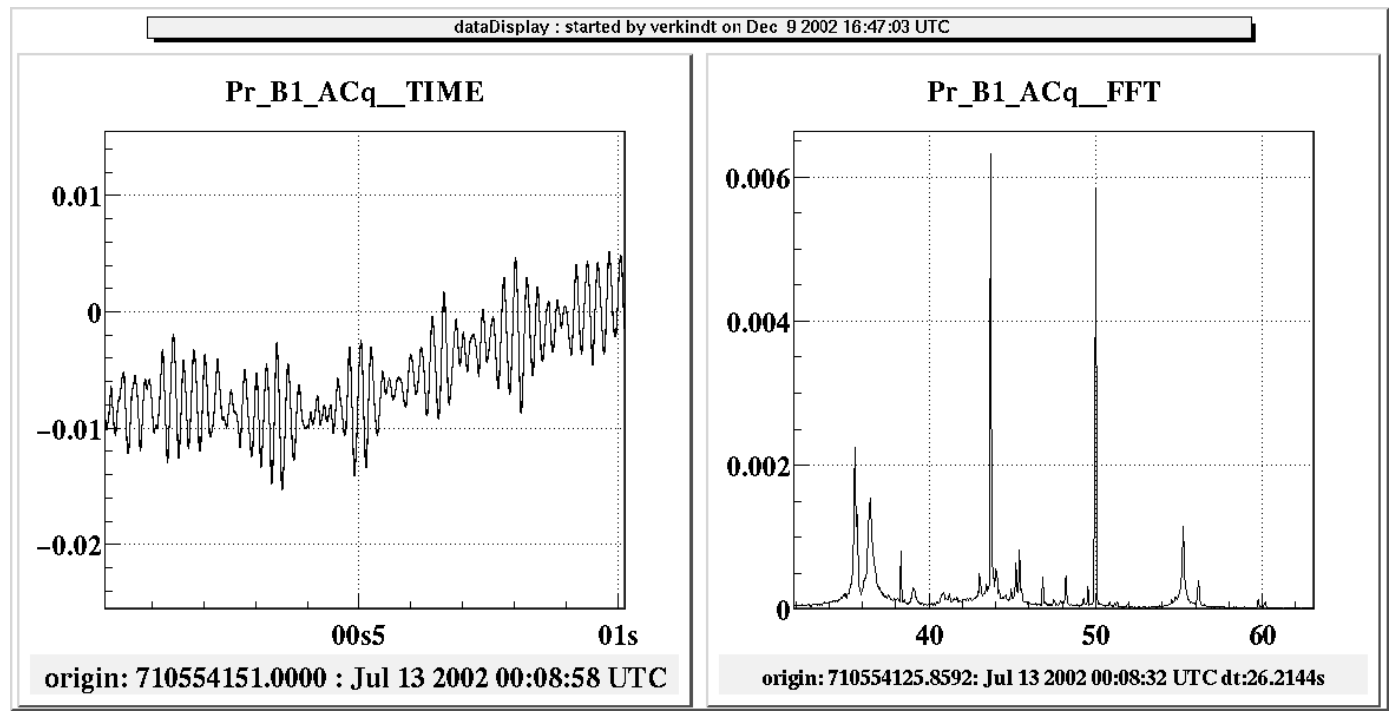

Figure 7. Time plot and spectrum plot of the dark fringe signal showing the beat phenomenon and the lines at $43.7 \mathrm{~Hz}$ and $50 \mathrm{~Hz}$. Vertical axis is in arbitrary units. Horizontal axis is time in seconds (0s8 mean 0.8 second).

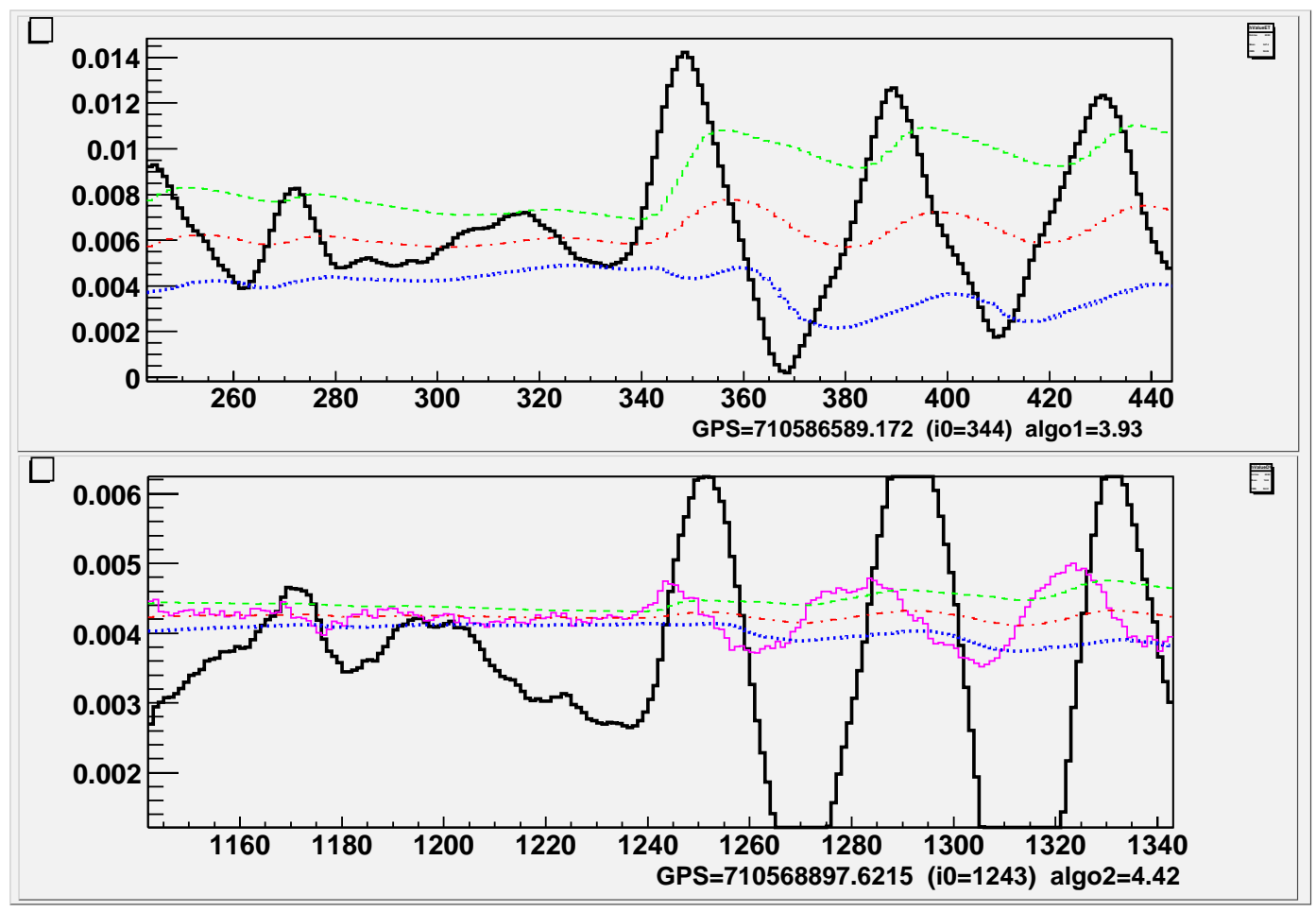

Figure 8. The largest events seen by algo1 and algo2, when data are down-sampled at 2kHz. [algo1 maximum: 3.93 at GPS=710586589.1720] [algo2 maximum: 4.42 at GPS=710568897.6215]. Colored lines have the same meaning as in figure 1. On upper plot, maximum of algo1 output is for sample 344. On lower plot, maximum of algo2 output is for sample 1243. Vertical axis is in arbitrary units. 


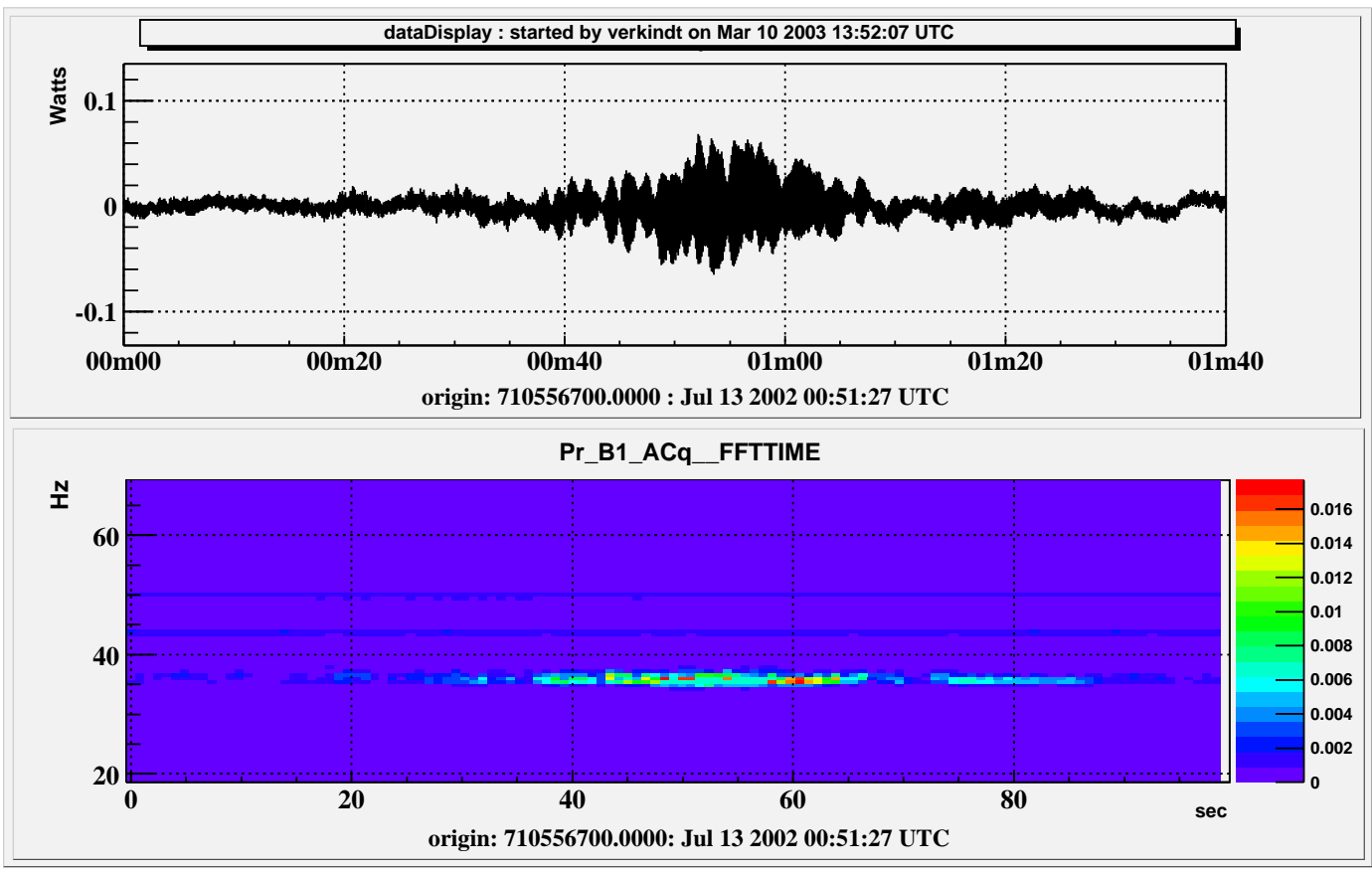

Figure 9. The 30 seconds of data with the $36 \mathrm{~Hz}$ noise, generating the main tail in the dark fringe signal distribution. Upper plot shows the B1 signals versus time. Its horizontal axis is time in minutes and seconds (0m40 mean 0 minutes and 40 seconds). Lower plot is the corresponding time-frequency plot. Its color scale is spectra amplitude in arbitrary units.

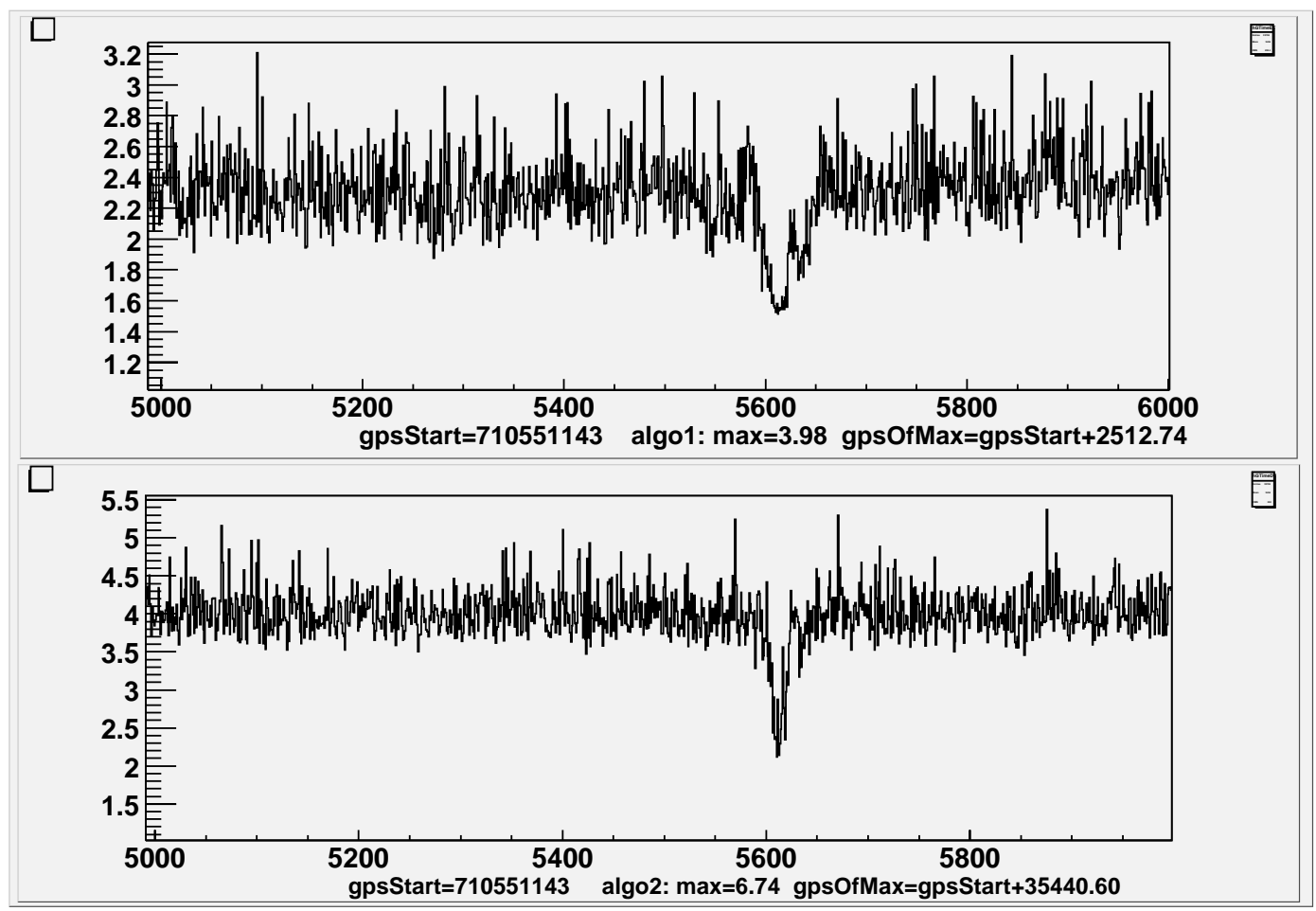

Figure 10. This is a zoom of the plots of figure 6. The 30 seconds of data with the $36 \mathrm{~Hz}$ noise are seen by the two algorithms as a minimum of their output. Upper plot is for algo1, lower plot is for algo2. Vertical axis is in arbitrary units. 